\title{
The C-C chemokine receptors CCR4 and CCR8 identify airway $T$ cells of allergen-challenged atopic asthmatics
}

\author{
Paola Panina-Bordignon, ${ }^{1}$ Alberto Papi, ${ }^{2}$ Margherita Mariani, ${ }^{1}$ Pietro Di Lucia, ${ }^{1}$ \\ Gianluca Casoni, ${ }^{2}$ Cinzia Bellettato, ${ }^{2}$ Cecilia Buonsanti, ${ }^{1}$ Deborah Miotto, ${ }^{3}$ \\ Cristina Mapp, ${ }^{3}$ Antonello Villa, ${ }^{4}$ Gianluigi Arrigoni, ${ }^{5}$ Leonardo M. Fabbri, ${ }^{6}$ \\ and Francesco Sinigaglia ${ }^{1}$ \\ ${ }^{1}$ Roche Milano Ricerche, Milano, Italy \\ ${ }^{2}$ Asthma and Chronic Obstructive Pulmonary Disease Research Center, and \\ ${ }^{3}$ Section of Occupational Medicine, Department of Clinical and Experimental Medicine, University of Ferrara, Ferrara, Italy \\ ${ }^{4}$ Department of Biological and Technological Research, and \\ ${ }^{5}$ Department of Pathology, San Raffaele Scientific Institute, Milano, Italy \\ ${ }^{6}$ Clinica di Malattie dell'Apparato Respiratorio, Universita' di Modena, Modena, Italy
}

Address correspondence to: Paola Panina-Bordignon, Roche Milano Ricerche, Via Olgettina 58, 20129 Milano, Italy.

Phone: 39-02-2884-804; Fax: 39-02-2153-203; E-mail: paola.panina@roche.com.

Paola Panina-Bordignon and Alberto Papi contributed equally to this work.

Received for publication March 2, 2001, and accepted in revised form May 1, 2001.

In vitro polarized human Th2 cells preferentially express the chemokine receptors CCR3, CCR4, and CCR8 and migrate to their ligands: eotaxin, monocyte-derived chemokine (MDC), thymus- and activation-regulated chemokine (TARC), and I-309. We have studied the expression of chemokines and chemokine receptors in the airway mucosa of atopic asthmatics. Immunofluorescent analysis of endobronchial biopsies from six asthmatics, taken 24 hours after allergen challenge, demonstrates that virtually all $\mathrm{T}$ cells express IL-4 and CCR 4 . CCR8 is coexpressed with CCR 4 on $28 \%$ of the T cells, while CCR3 is expressed on eosinophils but not on T cells. Expression of the CCR4-specific ligands MDC and TARC is strongly upregulated on airway epithelial cells upon allergen challenge, suggesting an involvement of this receptor/ligand axis in the regulation of lymphocyte recruitment into the asthmatic bronchi. In contrast to asthma, $T$ cells infiltrating the airways of patients with chronic obstructive pulmonary disease and pulmonary sarcoidosis produce IFN- $\gamma$ and express high levels of CXCR3, while lacking CCR4 and CCR8 expression. These data support the role of CCR4, of its ligands MDC and TARC, and of CCR8 in the pathogenesis of allergen-induced late asthmatic responses and suggest that these molecules could be considered as targets for therapeutic intervention.

J. Clin. Invest. 107:1357-1364 (2001).

\section{Introduction}

Trafficking of activated $\mathrm{T}$ cells into inflammatory sites is a tightly controlled process directed by multiple molecules, in particular adhesion molecules and chemokines $(1,2)$. Chemokines are small, secreted polypeptides that regulate lymphocyte trafficking by signaling through $G$ protein-coupled seven transmembrane receptors. A flurry of recent in vitro studies has demonstrated that Th1 and Th2 cells express distinct sets of chemokine receptors that might regulate the recruitment and localization of these cells to inflammatory sites $(3,4)$. CXCR3 and CCR5 have been associated with the Th 1 cytokine profile $(5,6)$, whereas CCR3, CCR4, and CCR8 have been associated with the Th2 phenotype (7-10). Selective recruitment of subsets of $\mathrm{CD}^{+}$effector $\mathrm{T}$ cells into sites of inflammation may contribute to the development of different pathological conditions.

$\mathrm{T}$ lymphocytes are involved in initiating and maintaining airway inflammation and obstruction in asthma (11). Activated $\mathrm{CD}^{+} \mathrm{T}$ cells producing IL-4, IL-5, and IL-13 have been identified in bronchoalveolar lavage and bronchial biopsies of both atopic and nonatopic asthmatic patients $(12,13)$, and they increase during late asthmatic responses (14-16). Despite the clear evidence for $\mathrm{T}$ cell involvement in asthma, the mechanisms of Th2 cell recruitment within the airways remain poorly defined. Recent studies have shown that the CCR3 ligand eotaxin is produced at high levels in human asthma and localizes to the airway epithelium and to inflammatory cells (17). Unlike other eosinophil-attracting chemokines, eotaxin binds to a single receptor, CCR3, that is highly expressed on eosinophils $(18,19)$. Because CCR3 is also expressed on basophils (20) and on in vitro polarized Th2 cells (7), it has been suggested that the sharing of CCR3 may allow these different cell types to colocalize at sites of eotaxin production. In addition to eotaxin, other chemokines have been shown to preferentially attract Th2 cells in vitro. These include monocyte-derived chemokine and I-309, binding to CCR4 and CCR8, respectively. Although these results suggest that these 
chemokines/chemokine receptor axes may be involved in the recruitment of Th2 lymphocytes that could lead to increased airway damage and altered physiology, very limited information is available on the expression and function of these specific receptors during the asthmatic inflammatory response.

In this study we demonstrate that the majority (>90\%) of T cells infiltrating the bronchial biopsies of allergen-challenged asthmatics produce IL- 4 and express CCR4. CCR8 was expressed on approximately $28 \%$ of infiltrating CCR $4^{+}$IL $-4^{+} \mathrm{T}$ cells. The two CCR 4 ligands, monocyte-derived chemokine (MDC) and thymus and activation-regulated chemokine (TARC), were expressed by airway epithelial cells and their expression was strongly upregulated after allergen challenge. A comparative analysis of $\mathrm{T}$ cells infiltrating the bronchi of patients with nonatopic inflammatory lung diseases such as chronic obstructive pulmonary disease (COPD) and pulmonary sarcoidosis revealed that this chemokine/chemokine receptor pattern was specific for atopic asthma. Unlike the T cells infiltrating the asthmatic samples, $\mathrm{T}$ cells in both COPD and sarcoidosis produce IFN- $\gamma$ and express CXCR3, while lacking CCR 4 and CCR8 expression. We conclude that CCR4 and, to a lesser extent, CCR8 mark the majority of $T$ cells infiltrating the airway of asthmatic patients after allergen challenge.

\section{Methods}

Subjects. Twenty-three subjects entered the study: twelve with atopic asthma, six with COPD, two with pulmonary sarcoidosis, and three healthy controls. All subjects were recruited from the outpatient clinic of the University of Ferrara. At the time of the study, patients with atopic asthma were in a stable condition and free from acute exacerbation of symptoms and from upper respiratory tract infections in the 4 months preceding the study. They had neither received oral/systemic steroids during the last 6 months nor inhaled corticosteroids during the last 6 weeks. Subjects were characterized by medical history, physical examination, and pulmonary function tests, including lung volumes, airway responsiveness to methacholine, or reversibility of airflow limitation, according to standardized techniques $(21,22)$. Predicted values of forced expiratory volume in 1 second $\left(\mathrm{FEV}_{1}\right)$ were derived from the European Community for Steel and Coal tables. All the subjects underwent chest $\mathrm{x}$-rays and bronchoscopy. The asthmatic patients were nonsmokers; all but one were males. The diagnosis of asthma was established on clinical and lung function measurements, according to the current international guidelines for diagnosis and treatment of asthma (23). They were all atopic as demonstrated by positive immediate skin prick test to at least one of ten common inhaled allergens. At the time of the study they had mild asthma (23) with normal lung function test and they were treated with inhaled salbutamol as needed. $\beta 2$-agonists were withheld for at least 12 hours before physiologic testing or allergen challenge. Among the asthmatics, six subjects underwent allergen challenge 24 hours before bronchoscopy. When (in one case) skin prick tests were positive to one or more antigens (skin wheal response $>5$ $\mathrm{mm}$ at 15 minutes), then the allergen selected for inhalation was determined by clinical history. Five subjects received grass pollens (Phleum pratensis, Phlpr) and one received house dust mites (Dermatophagoides pteronyssinus, Derpt). The other six asthmatics had similar skin test responsiveness (five reacted to grass pollens and one to house dust mites). They were exposed to allergen diluent (saline), via nebulizer as described below, 24 hours before bronchoscopy. All subjects were evaluated out of the respective allergen season or after attempts to minimize environmental exposure.

COPD was diagnosed according to the criteria recommended by the European Respiratory Society (24). The six patients (three of them males) were nonatopic and skin prick test-negative and had a history of chronic progressive symptoms, such as breathlessness, cough, and sputum production. They had no history of asthma or other allergic diseases (e.g., rhinitis). They were ex-smokers, and none of them had smoked in the 6 months preceding the study. They had chronic airflow limitation (forced expiratory volume measured after the first second expiration from total lung capacity $\left[\mathrm{FEV}_{1}\right]$, assessed by spirometry) of less than $80 \%$ predicted, with $\mathrm{FEV}_{1} /$ vital capacity less than $88 \%$ predicted (24). These parameters did not change significantly in the 5 months preceding the study.

Transbronchial biopsies were obtained from two male patients with active pulmonary sarcoidosis. The diagnosis of pulmonary sarcoidosis was based on the clinical presentation, the radiological findings, and the presence of noncaseating epithelioid cell granulomas in biopsy specimens from the lung, in the absence of other known causes of granulomatous disease (25). The two patients were nonsmokers, nonatopic with negative skin prick test, and had not received oral/systemic steroids during the last 6 months. Three normal subjects were accepted as controls, two of them males. They had normal physical examinations, chest x-rays, and lung function tests. The study was approved by the ethical committee of the University Hospital of Ferrara, and all patients gave their informed consent.

Methacholine challenge testing. Nonspecific airway responsiveness was assessed by methacholine inhalation challenge test using a Wright nebulizer. The test was performed by the 2 -minute tidal breathing dosing protocol (26). Results were expressed as the provoking concentration of methacholine required to decrease baseline $\mathrm{FEV}_{1}$ by $20 \%\left(\mathrm{PC}_{20}\right)$.

Allergen challenge. Allergen challenge was performed with an antigen that previously elicited a positive skin test response and that correlated with the clinical history of the patient, according to standardized protocols (27). With a De Vilbiss nebulizer, subjects inhaled five times to a total lung capacity, starting with an initial concentration of antigen of $1: 10^{6}$. Spirometry was per- 
formed before 5 and 15 minutes after the allergen inhalation challenge. Tenfold increasing concentrations were administered until a fall in $\mathrm{FEV}_{1}$ of $20 \%$ or more was achieved or a concentration of $1: 10^{2}$ was reached. The time of the fall in $\mathrm{FEV}_{1}$ of $20 \%$ or more (early asthmatic response, EAR) was recorded and labeled as time 0 . Spirometry was thereafter performed every 15 minutes for the first 2 hours and then hourly for 10 hours. After recovery from EAR, a subsequent fall in $\mathrm{FEV}_{1}$ greater than $20 \%$ base line was considered to be a late asthmatic response (LAR).

Bronchoscopy, bronchoalveolar lavage, and bronchial biopsies. Patients exposed to allergen/diluent challenge underwent bronchoscopy 24 hours after the exposure. Bronchoalveolar cells were obtained and analyzed as previously described (28). Three to five endobronchial biopsies were taken through a bronchoscope (Olympus BF type 1T10; Olympus Optical Co., Tokyo, Japan) with sterile forceps (FB 15C; Olympus Co.) from the subcarinae of basal segment bronchi of the right middle lobe (29).

Transbronchial lung biopsies. Alligator forceps were used via the bronchoscope to obtain four to five biopsies from the right lower lobe, according to standardized protocols (30).

Biopsies. Biopsies were embedded in Tissue-Tek II OCT (Miles Inc., Elkhart, Illinois, USA), frozen within 30 minutes in isopentane, precooled in liquid nitrogen, and processed as previously described (29).

Antibodies. Anti-human CD3 mouse mAb was from Novocastra Laboratories Ltd. (Newcastle upon Tyne, United Kingdom); anti-human EG2 mouse mAb was from Amersham Pharmacia Biotech (Cologno Monzese, Italy); anti-human I-309 goat polyclonal antibodies were from R\&D Systems Inc. (Minneapolis, Minnesota, USA) and Santa Cruz Biotechnology Inc. (Santa Cruz, California, USA). Rat anti-human CCR3, mouse anti-human CXCR3, goat and mouse anti-human IFN- $\gamma$, and goat anti-human IL-4 antibodies were from R\&D Systems Inc. Goat anti-human CCR4 antibody was from Santa Cruz Biotechnology Inc. Goat anti-human CCR8 antibody was from Alexis Corp., (San Diego, California, USA). Rabbit anti-human MDC and rabbit anti-human TARC antibodies were from Santa Cruz Biotechnology Inc. All the secondary antibodies, normal sera, avidin-biotin staining kit, peroxidase substrates, and mounting medium for immunohistochemistry were from Vector Laboratories Inc. (Burlingame, California, USA). All the conjugated and unconjugated secondary antibodies for immunofluorescence were from Jackson ImmunoResearch Laboratories Inc. (West Grove, Pennsylvania, USA).

Immunofluorescence. Immunofluorescent stainings were carried out on $5-\mu \mathrm{m}$-thick cryostat serial sections from each bronchial biopsy displaced in three adjacent series on slides. At least three series were used for each staining. Slides for double immunofluorescent stainings were postfixed with $4 \%$ paraformaldehyde in PBS, washed twice in PBS, and blocked first with $0.1 \%$ glycine/PBS and then with 15\% FBS/PBS (blocking solution). After five washes with wash buffer $(0.45 \mathrm{M} \mathrm{NaCl}, 0.24 \mathrm{M}$ $\mathrm{Na}_{2} \mathrm{HPO}_{4}, 0.24 \mathrm{M} \mathrm{NaH}_{2} \mathrm{PO}_{4}, 0.3 \%$ Triton X-100), the slides were incubated overnight at $4^{\circ} \mathrm{C}$ with the primary antibodies diluted in blocking buffer in a humidified chamber. The slides were washed again five times with wash buffer, and incubated with the fluorochrome-conjugated (Rhodamine Red-X- or Cy2-conjugated) secondary antibodies diluted in blocking buffer for 1 hour at room temperature. After five washes in wash buffer and two in PBS, the slides were mounted with $90 \%$ glycerol/PBS. Negative controls were performed omitting the primary antibody or using an isotype control antibody from the same species. Double labeling with primary antibodies from the same host species (CCR4CCR8 and CCR4-IL-4) was carried out as recommended by Vector Laboratories Inc. Briefly, after the first single staining, sections were incubated with $15 \%$ normal serum to saturate any open antigen-binding site on the Cy2-conjugated secondary antibody, so that binding of the other primary antibody was prevented. The slides were then incubated with an excess of unconjugated $\mathrm{Fab}$ secondary antibody to block any nonspecific binding site, and the other single staining was performed using a Rhodamine Red-X-conjugated secondary antibody. Control stainings using only one primary antibody and both secondary antibodies in every possible combination, and negative controls, omitting one or both primary antibodies, were performed. Once mounted, slides were analyzed with a confocal microscope (MRC-1024; Bio-Rad Laboratories Inc., Hercules, California, USA) equipped with a $15-\mathrm{mW}$ krypton/argon laser emitting at $488 \mathrm{~nm}$ and $647 \mathrm{~nm}$, mounted on a Nikon Eclipse E600 microscope (Nikon Instruments SpA, Sesto Fiorentino, Italy). Images were acquired and analyzed with Laser Sharp 3.2 software (Bio-Rad Laboratories Inc.).

Statistical analysis. Group data were expressed as means \pm SEM, or as median and interquartile range when appropriate. $\mathrm{PC}_{20} \mathrm{FEV}_{1}$ methacholine was expressed as geometric means (geometric standard error of the mean [GSEM]). Logarithmic transformation was done for this value in the statistical analysis. Differences between groups were analyzed using the Kruskal-Wallis test and the Mann-Whitney $U$ test when appropriate. Probability values of $P \leq 0.05$ were accepted as significant. With regard to immunofluorescence, at least three replicate measurements were performed by the same observer in nine randomly selected sections. The intraobserver reproducibility was assessed with the coefficient of variation and with the intraclass correlation coefficient. The intraobserver coefficient of variation was $7 \%$ and the intraobserver correlation coefficient was 0.71 .

\section{Results}

Th2 lymphocytes represent the predominant infiltrate in bronchial biopsies of atopic asthmatics after allergen challenge. Bronchial biopsies were obtained from six atopic asthmatics 24 hours after allergen challenge, six atopic asthmatics 24 hours after sham challenge 


\section{Figure 1}

Cytokine production by T lymphocytes that infiltrate the airway subepithelium of atopic asthmatics. Cryostat sections of a bronchial biopsy from a representative healthy subject ( $\mathbf{a}$ and $\mathbf{d}$ ), a representative sham-challenged atopic asthmatic ( $\mathbf{b}$ and $\mathbf{e}$ ), and a subject with atopic asthma 24 hours after allergen challenge ( $c$ and $\mathbf{f})$. Double immunofluorescent staining with antiCD3 (green) and anti-IL-4 (red) (a-c) or anti-IFN- $\gamma$ (red) $(\mathbf{d}-\mathbf{f})$ is shown. Arrows point to double-stained cells (yellow). Original magnification: $\times 40$. e, airway epithelium.

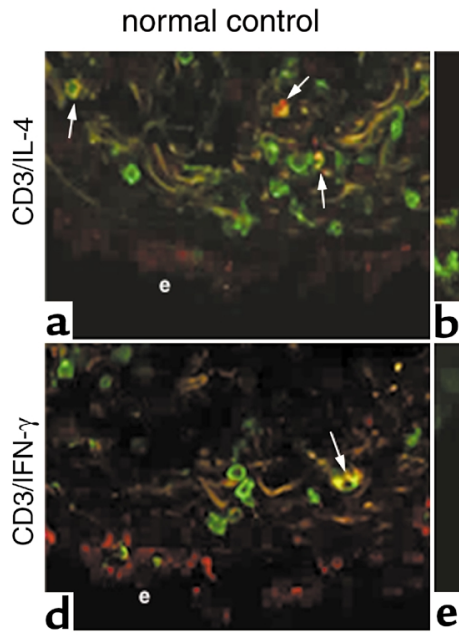

nonchallenged

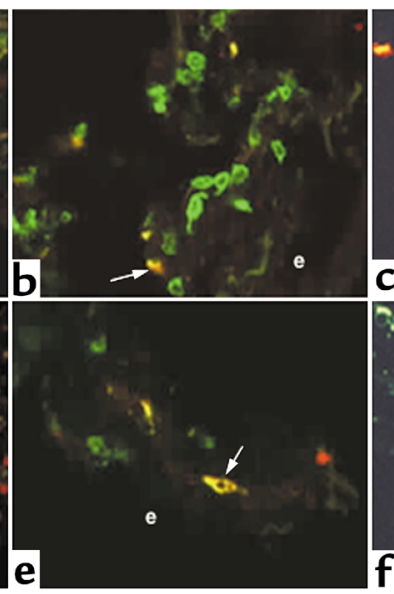

allergen-challenged

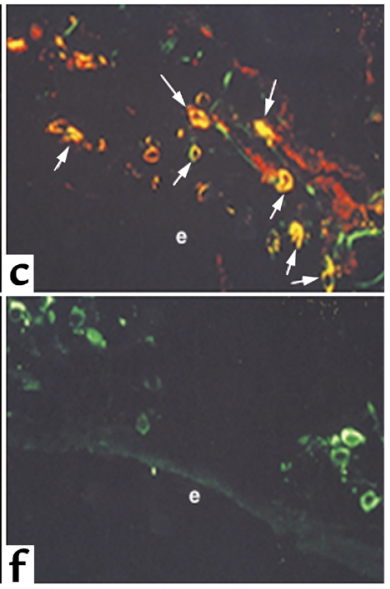

(nonsmokers; one female; mean age $27.6 \pm 2.8$; mean base-line $\mathrm{FEV}_{1} 98.5 \pm 4.5 ; \mathrm{PC}_{20} \mathrm{FEV}_{1}$ methacholine $1.3[0.9] \mathrm{mg} / \mathrm{ml}$ ), and three healthy controls (one female; mean age $39 \pm 6.3$ ). Each of the six asthmatic patients challenged with allergen developed both an EAR and an LAR (nonsmokers; two female; mean age $31.5 \pm 2.1$; mean base-line $\mathrm{FEV}_{1} 95.4 \pm$ 5.8; $\mathrm{PC}_{20} \mathrm{FEV}_{1}$ methacholine 1.5 [1.1] mg/ml). Bronchoalveolar eosinophils and lymphocytes were significantly increased at 24 hours after allergen challenge $(5 \%$ [3-5.25\%] vs. $1 \%[0-1.5 \%] ; P<0.01)$ and (3\% [2-6\%] vs. $1.5 \%$ [0.88-2\%]; $P<0.05)$.

Immunohistochemical analysis with anti-CD3, antiEG2, anti-CD14, anti-CD1a, and anti-tryptase antibodies revealed that only the number of $\mathrm{CD}^{+}$cells per square millimeter was significantly increased in the airway mucosa of sham-challenged asthmatics when compared with the nonasthmatic control group (874 [384-1480] vs. 274 [112-440], respectively; $P \leq 0.005)$. Upon allergen challenge, the number of subepithelial and intraepithelial $\mathrm{CD}^{+}$cells per square millimeter increased significantly (874 [384-1480] vs. 4400 [3870-7060], in sham-challenged and in allergen-challenged asthmatics, respectively; $P=0.0022$ ). The number of infiltrating eosinophils was not significantly increased at 24 hours after allergen challenge. At this time, cells staining positive for CD14, CD1a, or tryptase could hardly be detected in any of the tissues analyzed (data not shown). We then analyzed IL- 4 and IFN- $\gamma$ production in infiltrating $\mathrm{CD}^{+}$cells by double immunofluorescence. A significant proportion of $\mathrm{CD}^{+}$cells in healthy controls (34\%) and in sham-challenged asthmatics (40\%) produced IL-4, while virtually all the $\mathrm{CD}^{+}$ cells infiltrating the airway mucosa of allergen challenged asthmatics were IL-4+ (99.5\%) (Figure 1). By contrast, almost no IFN- $\gamma$-producing cells were present in the airway mucosa either after sham challenge $(1 \%$ among all the $\mathrm{CD}^{+}$cells) or allergen challenge $(0.5 \%$ among all the $\mathrm{CD}^{+}$cells). Few IFN- $\gamma$-producing cells were found in the airway mucosa of healthy controls ( $11 \%$ among all $\mathrm{CD}^{+}$cells). These results indicate a pre- dominant airway infiltration of Th2 cells at 24 hours after sham or allergen challenge.

$\mathrm{CD}^{+}$cells in bronchial biopsies of allergen-challenged atopic asthmatics express CCR4 and CCR8 but not CCR3. The selective migration of $\mathrm{CD}^{+}$cells to inflammation sites is a tightly regulated event depending upon the restricted expression of chemokine receptors on different Th cell subsets. We analyzed, by immunofluorescence, chemokine receptor expression on $\mathrm{T}$ cells infiltrating the airway mucosa. Since CCR3 is expressed on in vitro polarized Th2 cells, we first analyzed the tissue distribution of CCR3 expression in allergen-challenged and sham-challenged asthmatics. As shown in Figure 2, $\mathrm{CD}^{+}$cells infiltrating the bronchi of allergen-challenged asthmatics did not express CCR3. A few cells expressing CCR3 were only detectable in the airway mucosa of allergen-challenged asthmatics, not in sham-challenged asth-

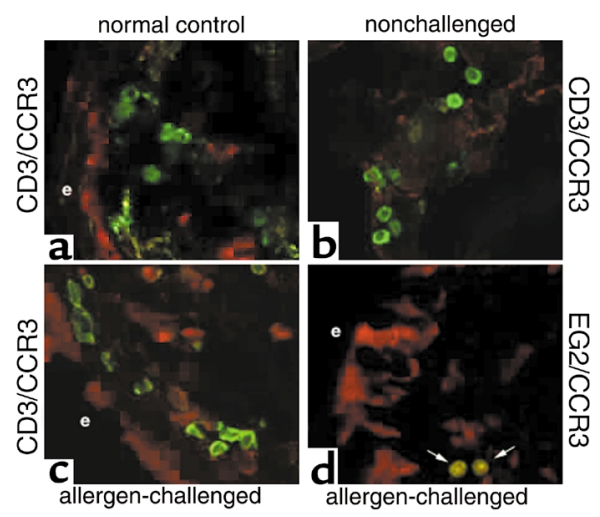

\section{Figure 2}

Expression of CCR3 by EG2 ${ }^{+}$cells and airway epithelial cells but not by $T$ cells infiltrating the airway of atopic asthmatics. Cryostat sections of bronchial biopsies from a representative healthy subject (a), a representative sham-challenged atopic asthmatic (b), and a representative subject with atopic asthma 24 hours after allergen challenge ( $\mathbf{c}$ and $\mathbf{d})$. (a-c) Double immunofluorescent staining with anti-CD3 (green) and anti-CCR3 (red). (d) Double immunofluorescent staining with antiEG2 (green) and anti-CCR3 (red). Arrows point to double-stained cells (yellow). Original magnification: $\times 40$. e, airway epithelium. 

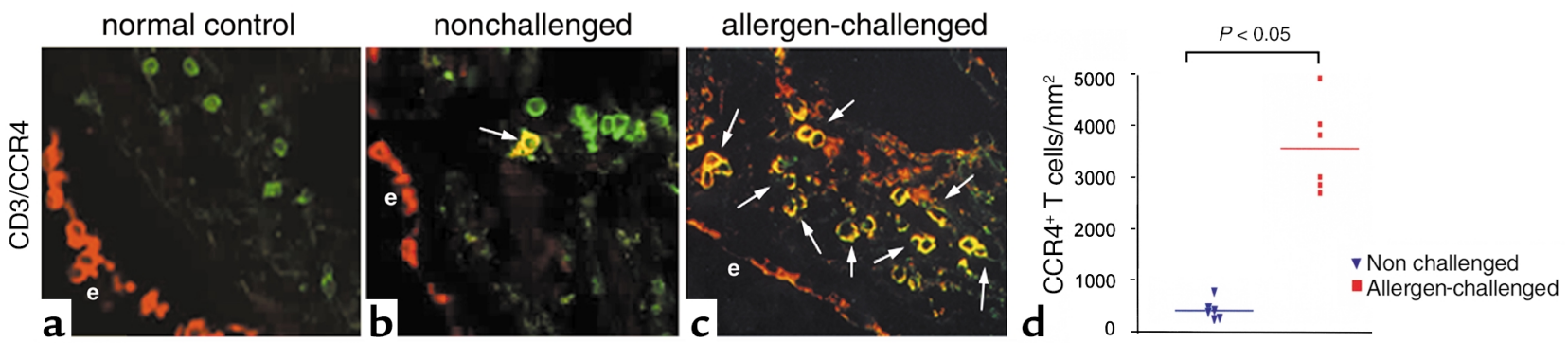

Figure 3

$(\mathbf{a}-\mathbf{c})$ Expression of CCR4 by T cells infiltrating the airway of atopic asthmatics. Cryostat sections of bronchial biopsies from a representative healthy subject (a), a representative sham-challenged atopic asthmatic (b), and a representative subject with atopic asthma 24 hours after allergen challenge (c). Double immunofluorescent staining with anti-CD3 (green) and anti-CCR4 (red) is shown. Arrows point to double-stained cells (yellow). Original magnification: $\times 40$. e, airway epithelium. (d) Individual counts of CCR4-expressing T lymphocytes in the airway subepithelium of atopic asthmatic patients 24 hours after allergen challenge $(n=6)$ compared with sham-challenged asthmatic subjects $(n=6)$. The horizontal bars indicate the mean value for each group ( $419 \pm 77 \mathrm{SE}, 3560 \pm 349 \mathrm{SE}$ in sham-challenged vs. allergen-challenged). The results are expressed as number of cells per square millimeter of subepithelium. Differences between groups were analyzed using the Mann-Whitney test.

matics or in healthy controls. These cells are likely to be eosinophils, as they coexpress the marker EG2.

The tissue distribution of CCR 4 expression in challenged asthmatics versus nonchallenged asthmatics and nonasthmatic controls is shown in Figure 3. Challenged asthmatics showed a significantly higher number of infiltrating CCR $4^{+}$Th2 cells per square millimeter compared with sham-challenged asthmatics (3410 [2790-4480] vs. 390 [261-606], respectively; $P=0.0022$ ). CCR 4 expression was also found on airway epithelial cells in all sections examined both in asthmatics and in controls (see Figures 2, 4, and 6).

$\mathrm{T}$ cells expressing CCR8 were also increased in bronchial biopsies from challenged asthmatics as compared with sham-challenged asthmatics (1070 [729-1160] vs. 251 [229-326], respectively; $P=0.0022$ ) and were undetectable in healthy controls (Figure 4). Double immunofluorescence staining with antiCCR4 and anti-CCR8 antibodies revealed that CCR8 ${ }^{+}$ Th2 cells represent a small subset $(28 \%)$ of the CCR $4^{+}$ Th2 population (Figure 5). Our data indicate that 24 hours after allergen challenge, the central airways of atopic asthmatics are infiltrated by Th2 cells that express CCR4, by a smaller population of Th2 cells coexpressing CCR4 and CCR8, and by a few eosinophils expressing CCR3. The maximal decrease in $\mathrm{FEV}_{1}$ during LAR positively correlated with the number of CCR $8^{+}$cells $\left(r^{2}=0.7361 ; P=0.0288\right)$ but not of CCR $4^{+}$cells $\left(r^{2}=0.2732 ; P=0.2874\right)$ infiltrating the airway mucosa.

$M D C$ and TARC are upregulated in airway epithelium after allergen challenge. On the same biopsies we also examined the expression of MDC and TARC. Both MDC and TARC expression was exclusively detected as a cytoplasmic staining in airway epithelial cells (Figure 6). While we did not observe any difference in the expression of MDC and TARC between healthy controls and sham-challenged asthmatics, their expression on airway epithelial cells was strongly upregulated upon allergen challenge.

Airway $\mathrm{CD}^{+}$cells of $\mathrm{COPD}$ and pulmonary sarcoidosis express CXCR3 but not CCR4. To evaluate the disease
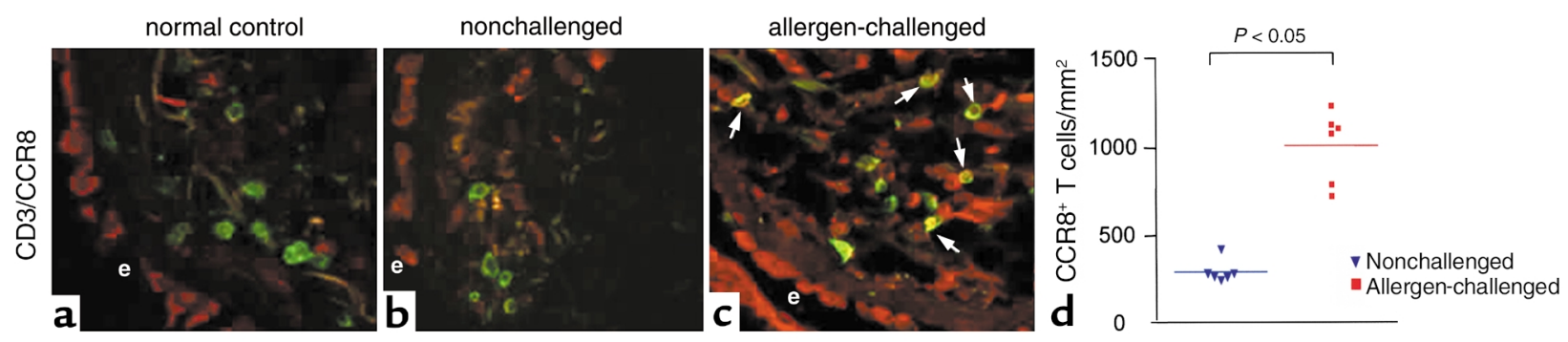

\section{Figure 4}

(a-c) Expression of CCR8 by T cells infiltrating the airway of atopic asthmatics. Cryostat sections of bronchial biopsies from a representative healthy subject (a), a representative sham-challenged atopic asthmatic (b), and a representative subject with atopic asthma 24 hours after allergen challenge (c). Double immunofluorescent staining with anti-CD3 (green) and anti-CCR8 (red) is shown. Arrows point to double-stained cells (yellow). Original magnification: $\times 40$. e, airway epithelium. (d) Individual counts of CCR8-expressing T lymphocytes in the airway subepithelium of atopic asthmatic patients 24 hours after allergen challenge $(n=6)$ compared with shamchallenged asthmatic subjects $(n=6)$. The horizontal bars indicate the mean value for each group $(269 \pm 25$ SE and $984 \pm 84$ SE in sham-challenged vs. allergen-challenged). The results are expressed as number of cells per square millimeter of subepithelium. Differences between groups were analyzed using the Mann-Whitney test. 


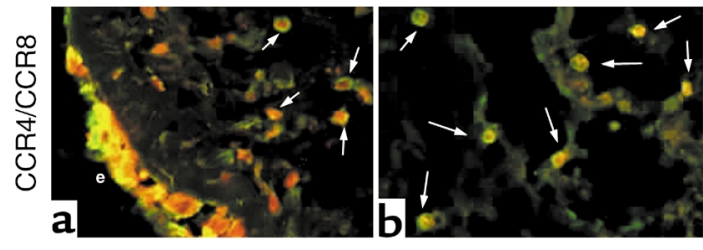

Figure 5

Coexpression of CCR 4 and CCR8 by infiltrating T cells ( $\mathbf{a}$ and $\mathbf{b}$ ) and airway epithelial cells (a) of atopic asthmatics. Double immunofluorescent staining with anti-CCR8 (green) and anti-CCR4 (red) on a bronchial biopsy from a representative subject with atopic asthma 24 hours after allergen challenge. Arrows indicate double positive Tlymphocytes (yellow). Original magnification: $\times 40$. e, airway epithelium.

specificity of the CCR4/CCR8 expression pattern, we analyzed chemokine receptor expression in bronchial biopsies from patients with COPD and pulmonary sarcoidosis. COPD is a nonatopic inflammatory process of the airway mucosa and lung parenchyma resulting in a chronic airflow limitation (31). Double immunofluorescent analysis with anti-IFN- $\gamma /$ antiCD3, anti-IL-4/anti-CD3, anti-CCR4/anti-CD3, antiCCR8/anti-CD3 (data not shown), and anti-IFN- $\gamma /$ anti-CXCR3 or anti-IL-4/anti-CCR4 (Figure 7) antibodies was performed on bronchial biopsies from six allergen-challenged asthmatic patients and six patients with COPD, and on transbronchial biopsies from two patients with active pulmonary sarcoidosis. In contrast to asthma patients (Figure 7, a and d), $\mathrm{T}$ cells infiltrating the airways of COPD patients produce IFN- $\gamma$ and express the CXCR3 chemokine receptor, while lacking expression of IL-4, CCR4 (Figure 7, $\mathrm{b}$ and e), and CCR8 (data not shown). IL-4, CCR4, and CCR8 expressions were also not detected on lung $\mathrm{T}$ cells from pulmonary sarcoidosis (Figure $7 \mathrm{c}$ and data not shown), a disease characterized by a typical cell-mediated Th1-type inflammatory response (28, 32-34). The Th1 nature of $\mathrm{T}$ cells in pulmonary sarcoidosis was confirmed by IFN- $\gamma$ expression. Furthermore, Th1 cells surrounding the granuloma express CXCR3 (Figure 7f).

\section{Discussion}

In this study we found that the $T$ cells infiltrating the airway mucosa of atopic asthmatics have a Th2 phenotype, that they express the chemokine receptors CCR4 and, to a lesser extent, CCR8, and that their number increases after allergen challenge. Interestingly, we found a correlation between the number of CCR $8^{+} \mathrm{Th} 2$ cells and the severity of the asthmatic response to allergen challenge. Finally, we observed that MDC and TARC are expressed by airway epithelial cells of healthy controls and stable asthmatics. Furthermore, their expression is strongly upregulated after allergen challenge.

Effector $\mathrm{T}$ helper cells are polarized with respect to their chemokine receptor expression as well as their cytokine production, and in vitro polarized Th2 cells preferentially express CCR3, CCR4, and CCR8. Our studies demonstrate that after allergen challenge, virtually all $\mathrm{T}$ cells infiltrating the airways produce IL-4 and express CCR4 but not CCR3. The expression of CCR 4 was paralleled by strong expression of its ligands MDC and TARC in the airway epithelial cells after allergen challenge, suggesting that the CCR4/ligands axis may be involved in the Th2 cell recruitment. Recent evidence indicates that IL-4 in combination with TNF- $\alpha$ upregulates TARC production by an airway epithelial cell line (35). Thus, it is tempting to speculate that the CCR4 ligands induced by IL-4 in Th2 diseases such as asthma chemoattract CCR4 ${ }^{+}$ $\mathrm{T}$ cells, which in turn are induced to express more IL-4, establishing a mechanism for amplifying Th2 responses (36). Collectively, these data support the role of the CCR4/ligands axis in the pathogenesis of inflammation and tissue damage in asthma. Studies in a mouse model of antigen-induced airway inflammation have indeed provided evidence for a crucial role of the CCR4/MDC and CCR4/TARC axes in the recruitment of Th2 cells into the lung $(37,38)$. Repeated allergen challenge resulted in an increased frequency of CCR4expressing Th2 cells in the inflamed airways, and antiMDC as well as anti-TARC antibodies suppressed cell influx and airway hyperresponsiveness $(38,39)$. Surprisingly, CCR4-deficient mice are still capable of developing allergic airway inflammation (40). Since CCR4 and its ligands have been implicated in thymocyte development (41), it is possible that CCR4-deficient $\mathrm{T}$ cells from gene-ablated mice upregulate some compensatory mechanisms. Recent data concerning the upregulation of CCR4 in vivo have also been obtained in atopic dermatitis (AD) patients (42). $\mathrm{AD}$ is a disease in which the lymphocytes invading the skin are, at least

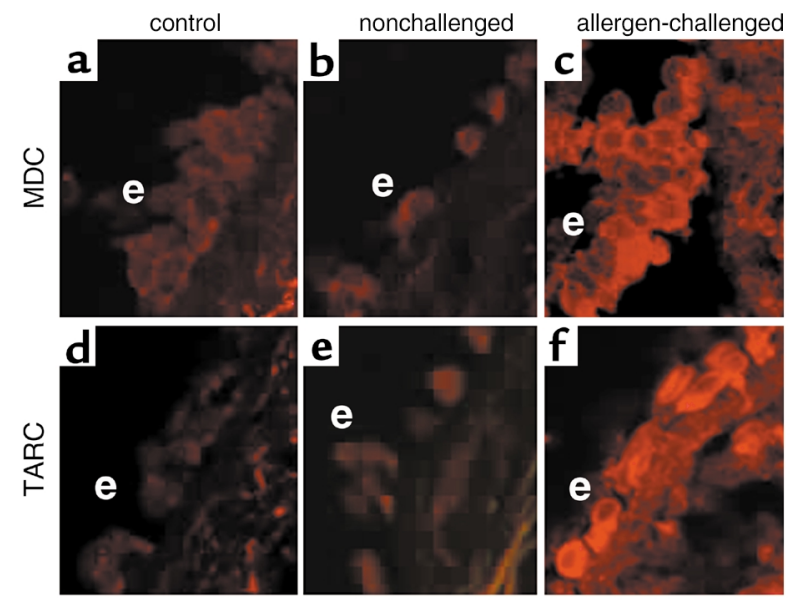

\section{Figure 6}

Upregulation of MDC and TARC expression on airway epithelial cells of allergen-challenged atopic asthmatics. Cryostat sections of bronchial biopsies from a representative healthy subject ( $\mathbf{a}$ and $\mathbf{d}$ ), a representative sham-challenged atopic asthmatic ( $\mathbf{b}$ and $\mathbf{e}$ ), and a representative subject with allergic asthma 24 hours after allergen challenge (c and $\mathbf{f}$ ). Immunofluorescent staining with anti$\operatorname{MDC}(\mathbf{a}-\mathbf{c})$ and anti-TARC $(\mathbf{d}-\mathbf{f})$ is shown. Original magnification: $\times 40$. e, airway epithelium. 


\section{Figure 7}

Coexpression of CCR4/IL-4 and CXCR3/IFN- $\gamma$ by lymphocytes infiltrating the airway in allergic asthma, COPD, and pulmonary sarcoidosis. Cryostat sections of bronchial biopsies from a representative subject with allergic asthma 24 hours after allergen challenge (a and $\mathbf{d}$ ), and from a representative subject with COPD ( $\mathbf{b}$ and $\mathbf{e})$. Cryostat sections of transbronchial biopsy from a representative subject with pulmonary sarcoidosis ( $\mathbf{c}$ and $\mathbf{f}$ ). Double immunofluorescent staining with anti-CCR4 (red) and anti-IL-4 (green) (a-c), and with anti-CXCR3 (green) and anti-IFN- $\gamma$ (red) (d-f), is shown. Arrows point to double positive T lymphocytes. Original magnification: $\times 40$. e, bronchial epithelium. challenged asthmatic
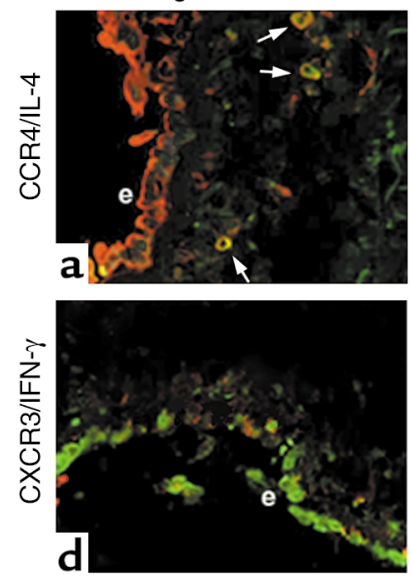

COPD

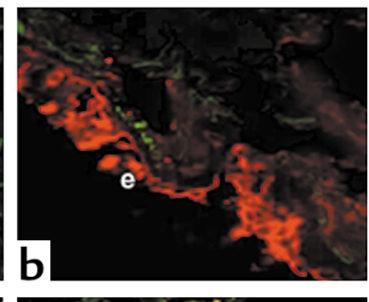

sarcoidosis

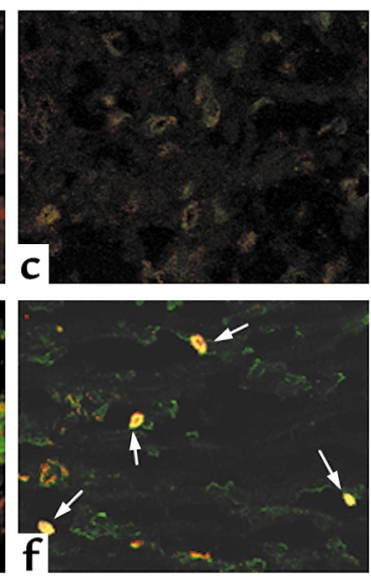

in the initial phase, Th2-type cells. Expression of the CCR4 ligand MDC was found in both dendritic cells and Th2 cells in the skin of AD patients (43). Furthermore, in a mouse model of bacteria-induced fulminant hepatitis, in which Th2 cells are the cause of liver injury after LPS administration, CCR4 was found preferentially expressed in IL-4-producing Th2 cells in vivo. Neutralization of the CCR4 ligand TARC decreased IL-4 production by liver-infiltrating T cells (44).

CCR8 was expressed, after allergen challenge, in approximately $28 \%$ of the CCR $4 / \mathrm{IL}-4$ double positive airway $\mathrm{T}$ cells. To the best of our knowledge this is the first report of CCR8 expression on $\mathrm{T}$ cells in a human pathology. The functional role of this $\mathrm{T}$ cell population is currently unclear; however, it was recently reported that the deletion of CCR8 attenuates eosinophil accumulation, Th2 cytokine production, and airway hyperresponsiveness in a cockroach allergen model of asthma (45). Interestingly, we found that the number of CCR $8^{+} \mathrm{Th} 2$ cells infiltrating the airway mucosa in challenged asthmatics correlates with the degree of airflow limitation during LAR. The precise mechanism by which CCR $8^{+}$Th 2 cells are linked to airflow limitation needs to be further investigated both in vitro and in vivo. However, our data suggest that a CCR8 selectively oriented recruitment of Th2 cells into the airways may be involved in the development of airflow limitation after allergen exposure. Surprisingly, we could not find expression of the CCR8-specific ligand I-309 either in the airway mucosa or in epithelial cells. TARC, whose expression is strongly upregulated by airway epithelial cells after allergen challenge, could serve, in vivo, as a ligand for CCR8, although this possibility is debatable $(46,47)$. An additional possibility is that CCR $8^{+}$cells in the airway mucosa respond to a yet unidentified ligand.

In the present study of bronchial biopsies from atopic asthmatics we show that CCR 3 is not expressed by airway-infiltrating $\mathrm{T}$ cells either before or after allergen challenge. These data are in agreement with previous observations reporting in vivo expression of CCR 3 on eosinophils and basophils but not on T cells in the skin of patients with systemic sclerosis (48). Interestingly, in a mouse model of airway hyperresponsiveness, expression of CCR3 was transient and was found only during the initial aeroallergen challenge (37). This may be explained by the observation that while in the mouse CCR3 is upregulated by anti-CD3 stimulation, in human $\mathrm{T}$ cells TCR-mediated activation results in downregulation of CCR3 (49).

The comparative analysis of chemokine receptor expression in a different airway disease, such as COPD, clearly indicated that CCR4 expression is specific to asthma. COPD is a disease characterized by the progressive development of airflow limitation associated with a chronic inflammatory process that differs from that seen in asthma, with different mediators, inflammatory effects, and response to treatment (31). In contrast to asthma, $\mathrm{T}$ cells in the bronchial mucosa of COPD patients predominantly express IFN- $\gamma$ and CXCR3 but do not express IL-4 and CCR4. The lack of IL-4 expression by infiltrating $\mathrm{T}$ cells confirms the nonatopic nature of COPD, while the expression of CXCR3 is in agreement with a number of published in vitro and in vivo studies in which CXCR3 was consistently found associated with Th1-type responses $(5,6$, 50). Similarly, in sarcoidosis, a disease characterized by a typical cell-mediated Th1-type inflammatory response $(25,28,32-34)$, we describe that lung T cells express IFN- $\gamma$ and CXCR3 but not IL-4 and CCR4. These results are consistent with a previous report documenting CXCR3 expression on $\mathrm{CD} 4^{+} \mathrm{T}$ cells from the bronchoalveolar lavage of sarcoidosis patients (51).

In conclusion, we have demonstrated that upon allergen challenge, CCR4 and to a lesser extent CCR8 are strongly expressed by $\mathrm{T}$ cells in airway mucosa, and their ligands TARC and MDC are upregulated in airway epithelial cells of atopic asthmatics. The coexpression of these chemokines and their receptors indicate their involvement in the regulation of lymphocyte recruitment into the asthmatic lung, suggesting that neutralization of this pathway in vivo may modify $\mathrm{T}$ cell migration into the airways and the consequent disease progression. 


\section{Acknowledgments}

We thank Maura Francolini for help with the confocal analysis, Lorenzo Corbetta and Franco Ravenna for performing bronchoscopies, and Daniele D'Ambrosio and Luciano Adorini for the critical reading of this manuscript. The work was supported by grants from the Italian Ministry of University and Research (MURST), Associazione per la Ricerca e la Cura dell'Asma, and Consorzio Ferrararicerche.

1. Butcher, E.C., and Picker, L.J. 1996. Lymphocyte homing and homeostasis. Science. 272:60-66.

2. Rossi, D., and Zlotnik, A. 2000. The biology of chemokines and their receptors. Annu. Rev. Immunol. 18:217-242.

3. Bonecchi, R., et al. 1998. Differential expression of chemokine receptors and chemotactic responsiveness of type $1 \mathrm{~T}$ helper cells (Th1s) and Th2s. J. Exp. Med. 187:129-134.

4. Sallusto, F., Lenig, D., Mackay, C.R., and Lanzavecchia, A. 1998. Flexible programs of chemokine receptor expression on human polarized Thelper 1 and 2 lymphocytes. J. Exp. Med. 187:875-883.

5. Loetscher, P., et al. 1998. CCR5 is characteristic of Th1 lymphocytes. Nature. 391:344-345.

6. Qin, S., et al. 1998. The chemokine receptor CXCR3 and CCR5 mark subsets of T cell associated with certain inflammatory reactions. J. Clin. Invest. 101:746-754.

7. Sallusto, F., Mackay, C., and Lanzavecchia, A. 1997. Selective expression of the eotaxin receptor CCR3 by human T helper 2 cells. Science. 277:2005-2007.

8. Gerber, B.O., et al. 1997. Functional expression of the eotaxin receptor CCR3 in $\mathrm{T}$ lymphocytes co-localizing with eosinophils. Curr. Biol. 7:836-843.

9. Imai, T., et al. 1999. Selective recruitment of CCR4-bearing Th2 cells toward antigen presenting cells by the CC chemokine thymus and activation regulated chemokine and macrophage-derived chemokine. Int. Immunol. 11:81-88.

10. Zingoni, A., et al. 1998. The chemokine receptor CCR8 is preferentially expressed in Th2 but not Th1 cells. J. Immunol. 161:547-551.

11. Kon, O.M., and Kay, A.B. 1999. T cells and chronic asthma. Int. Arch. Allergy Immunol. 118:133-135.

12. Ying, S., et al. 1997. Expression of IL-4 and IL-5 mRNA and protein product by CD4+ and CD8+ T cells, eosinophils and mast cells in bronchial biopsies obtained from atopic and nonatopic (intrinsic) asthmatics. $J$. Immunol. 158:3539-3544.

13. Robinson, D.S., et al. 1992. Predominant Th2-type bronchoalveolar lavage T-lymphocyte population in atopic asthma. N. Engl. J. Med. 326:298-304

14. Bentley, A.M., et al. 1993. Increases in activated T lymphocytes, eosinophils, and cytokine messenger RNA expression or IL-5 and GM-CSF in bronchial biopsies after allergen inhalation challenge in atopic asthmatics. Am. J. Respir. Cell. Mol. Biol. 8:35-42.

15. Del Prete, G.F., et al. 1993. Allergen exposure induces the activation of allergen-specific Th2 cells in the airway mucosa of patients with allergic respiratory disorders. Eur. J. Immunol. 23:1445-1449.

16. Bodey, K.J., et al. 1999. Cytokine profiles of BAL T cells and T-cell clones obtained from human asthmatic airways after local allergen challenge. Allergy. 54:1083-1093.

17. Lamkhioued, B., et al. 1997. Increased expression of eotaxin in bronchoalveolar lavage and airways of asthmatics contributes to the chemotaxis of eosinophils to the site of inflammation. J. Immunol. 159:4593-4601.

18. Ponath, P.D., et al. 1996. Molecular cloning and characterization of a human eotaxin receptor expressed selectively on eosinophils. J. Exp. Med. 183:2437-2448.

19. Daugherty, B.L., et al. 1996. Cloning, expression, and characterization of the human eosinophil eotaxin receptor. J. Exp. Med. 183:2349-2354.

20. Uguccioni, M., et al. 1997. High expression of the chemokine receptor CCR3 in human blood basophils. Role in activation by eotaxin, MCP-4, and other chemokines. J. Clin. Invest. 100:1137-1143.

21. Quanjer, P.H., et al. 1993. Symbols, abbreviations and units. Working Party Standardization of Lung Function Tests, European Community for Steel and Coal. Eur. Respir. J. Suppl. 16:85-100.

22. Crapo, R.O., et al. 2000. Guidelines for methacholine and exercise challenge testing-1999. This official statement of the American Thoracic Society was adopted by the ATS Board of Directors, July 1999. Am. J. Respir. Crit. Care Med. 161:309-329.

23. Sheffer, A.L. 1995. Global initiative for asthma. NHLBI/WHO report. NIH. Bethesda, Maryland, USA. 2-8.

24. Siafakas, N.M., et al. 1995. Optimal assessment and management of chronic obstructive pulmonary disease (COPD). The European Respira- tory Society Task Force. Eur. Respir. J. 8:1398-1420.

25. 1999. Statement on sarcoidosis. Joint Statement of the American Thoracic Society (ATS), the European Respiratory Society (ERS) and the World Association of Sarcoidosis and Other Granulomatous Disorders (WASOG) adopted by the ATS Board of Directors and by the ERS Executive Committee, February 1999. Am. J. Respir. Crit. Care Med. 160:736-755.

26. Sterk, P.J., et al. 1993. Airway responsiveness. Standardized challenge testing with pharmacological, physical and sensitizing stimuli in adults. Report Working Party Standardization of Lung Function Tests, European Community for Steel and Coal. Official Statement of the European Respiratory Society. Eur. Respir. J. Suppl. 16:53-83.

27. Smith, H.R., et al. 1992. Inflammatory cells and eicosanoid mediators in subjects with late asthmatic responses and increases in airway responsiveness. J. Allergy Clin. Immunol. 89:1076-1084.

28. Rogge, L., et al. 1999. Antibodies to the IL-12 receptor beta 2 chain mark human Th1 but not Th2 cells in vitro and in vivo. J. Immunol. 162:3926-3932

29. Di Stefano, A., et al. 1996. Airflow limitation in chronic bronchitis is associated with T-lymphocyte and macrophage infiltration of the bronchial mucosa. Am. J. Respir. Crit. Care Med. 153:629-632.

30. McDouglas, J.C., and Cortese, D.A. 1994. Bronchoscopic lung biopsy. In Broncoscopy. U.B.S. Prakash, editor. Raven Press. New York, New York, USA 141-146.

31. Barnes, P.J. 2000. Chronic obstructive pulmonary disease. N. Engl. J. Med. 343:269-280.

32. Minshall, E.M., et al. 1997. Cytokine mRNA gene expression in active and nonactive pulmonary sarcoidosis. Eur. Respir. J. 10:2034-2039.

33. Inui, N., Chida, K., Suda, T., and Nakamura, H. 2001. TH1/TH2 and TC1/TC2 profiles in peripheral blood and bronchoalveolar lavage fluid cells in pulmonary sarcoidosis. J. Allergy Clin. Immunol. 107:337-344.

34. Wahlstrom, J., et al. 2001. Analysis of intracellular cytokines in cd4(+) and cd8(+) lung and blood t cells in sarcoidosis. Am. J. Respir. Crit. Care Med. 163:115-121.

35. Sekiya, T., et al. 2000. Inducible expression of a Th2-type CC chemokine thymus- and activation-regulated chemokine by human bronchial epithelial cells. J. Immunol. 165:2205-2213.

36. Bonecchi, R., et al. 1998. Divergent effects of interleukin-4 and interferongamma on macrophage-derived chemokine production: an amplification circuit of polarized T helper 2 responses. Blood. 92:2668-2671.

37. Lloyd, C.M., et al. 2000. CC chemokine receptor (CCR)3/eotaxin is followed by CCR $4 /$ monocyte-derived chemokine in mediating pulmonary $\mathrm{T}$ helper lymphocye type 2 recruitment after serial antigen challenge in vivo. J. Exp. Med. 191:265-274.

38. Kawasaki, S., et al. 2001. Intervention of thymus and activation-regulated chemokine attenuates the development of allergic airway inflammation and hyperresponsiveness in mice. J. Immunol. 166:2055-2062.

39. Gonzalo, J.-A., et al. 1999. Mouse monocyte-derived chemokine is involved in airway hyperreactivity and lung inflammation. J. Immunol. 163:403-411.

40. Chvatchko, Y., et al. 2000. A key role for CC chemokine receptor 4 in lipopolysaccharide-induced endotoxic shock. J. Exp. Med. 191:1755-1764.

41. Campbell, J.J., Pan, J., and Butcher, E.C. 1999. Cutting edge: developmental switches in chemokine responses during $\mathrm{T}$ cell maturation. J. Immunol. 163:2353-2357.

42. Vestergaard, C., et al. 2000. A Th2 chemokine, TARC, produced by keratinocytes may recruit CLA+CCR4+ lymphocytes into lesional atopic dermatitis skin. J. Invest. Dermatol. 115:640-646.

43. Galli, G., et al. 2000. Macrophage-derived chemokine production by activated human $T$ cells in vitro and in vivo: preferential association with the production of type 2 cytokines. Eur. J. Immunol. 30:204-210.

44. Yoneyama, H., et al. 1998. Pivotal role of TARC, a CC chemokine, in bacteria-induced fulminant hepatic failure in mice. J. Clin. Invest. 102:1933-1941.

45. Chensue, S.W., et al. 2001. Aberrant in vivo T helper type 2 cell response and impaired eosinophil recruitment in CC chemokine receptor 8 knockout mice. J. Exp. Med. 193:573-584.

46. Bernardini, G., et al. 1998. Identification of the CC chemokines TARC and macrophage inflammatory protein-1 beta as novel functional ligands for the CCR8 receptor. Eur. J. Immunol. 28:582-588.

47. Garlisi, C.G., et al. 1999. The assignment of chemokine-chemokine receptor pairs: TARC and MIP-1 beta are not ligands for human CC-chemokine receptor 8. Eur. J. Immunol. 29:3210-3215.

48. Annunziato, F., et al. 1999. Assessment of chemokine receptor expression by human Th1 and Th2 cells in vitro and in vivo. J. Leukoc. Biol. 65:691-699.

49. D'Ambrosio, D., et al. 1998. Selective up-regulation of chemokine receptors CCR 4 and CCR8 upon activation of polarized human type 2 Th cells. J. Immunol. 161:5111-5115.

50. Shields, P.L., et al. 1999. Chemokine and chemokine receptor interactions provide a mechanism for selective $T$ cell recruitment to specific liver compartments within hepatitis C-infected liver. J. Immunol. 163:6236-6243.

51. Agostini, C., et al. 1998. Involvement of the IP-10 chemokine in sarcoid granulomatous reactions. J. Immunol. 161:6413-6420. 